\title{
Molecular Insights into How the Dimetal Center in Dihydropyrimidinase Can Bind the Thymine Antagonist 5-Aminouracil: A Different Binding Mode from the Anticancer Drug 5-Fluorouracil
}

\author{
En-Shyh Lin $\mathbb{D}^{1},{ }^{1}$ Ren-Hong Luo, ${ }^{2}$ Ya-Ching Yang, ${ }^{2}$ and Cheng-Yang Huang $\mathbb{D}^{2,3}$ \\ ${ }^{1}$ Department of Beauty Science, National Taichung University of Science and Technology, Taichung City, Taiwan \\ ${ }^{2}$ School of Biomedical Sciences, Chung Shan Medical University, Taichung City, Taiwan \\ ${ }^{3}$ Department of Medical Research, Chung Shan Medical University Hospital, Taichung City, Taiwan
}

Correspondence should be addressed to Cheng-Yang Huang; cyhuang@csmu.edu.tw

Received 3 November 2021; Accepted 27 January 2022; Published 14 February 2022

Academic Editor: Jamal Rafique

Copyright (C) 2022 En-Shyh Lin et al. This is an open access article distributed under the Creative Commons Attribution License, which permits unrestricted use, distribution, and reproduction in any medium, provided the original work is properly cited.

Dihydropyrimidinase (DHPase) is a key enzyme for pyrimidine degradation. DHPase contains a binuclear metal center in which two $\mathrm{Zn}$ ions are bridged by a posttranslationally carbamylated lysine. DHPase catalyzes the hydrolysis of dihydrouracil to $\mathrm{N}$ carbamoyl- $\beta$-alanine. Whether 5-aminouracil (5-AU), a thymine antagonist and an anticancer drug that can block DNA synthesis and induce replication stress, can interact with DHPase remains to be investigated. In this study, we determined the crystal structure of Pseudomonas aeruginosa DHPase (PaDHPase) complexed with 5-AU at $2.1 \AA$ resolution (PDB entry 7E3U). This complexed structure revealed that 5-AU interacts with $\mathrm{Zn} \alpha$ (3.2 $⿱$ A), $\mathrm{Zn} \beta$ (3.0 $\mathrm{\AA}$ ), the main chains of residues Ser289 (2.8 $\mathrm{\AA}$ ) and Asn337 (3.3 $\AA$ ), and the side chain of residue Tyr155 (2.8 $)$. These residues are also known as the substrate-binding sites of DHPase. Dynamic loop I (amino acid residues Pro65-Val70) in PaDHPase is not involved in the binding of 5-AU. The fluorescence quenching analysis and site-directed mutagenesis were used to confirm the binding mode revealed by the complexed crystal structure. The 5-AU binding mode of PaDHPase is, however, different from that of 5-fluorouracil, the best-known fluoropyrimidine used for anticancer therapy. These results provide molecular insights that may facilitate the development of new inhibitors targeting DHPase and constitute the 5-AU interactome.

\section{Introduction}

Pyrimidine bases are essential for the replication of genetic information, cellular metabolism, and cell growth in all biological systems [1]. Uracil derivatives, especially 5substituted uracils, play a significant role in pharmacological activities, such as antiviral [2], anticancer drugs [3], antibacterial [4], anti-inflammatory $[5,6]$, and antitumor activities [7-9]. For example, 5-fluorouracil (5-FU) is an FDAapproved drug with a remarkable therapeutic effect for the systemic treatment of cancers of the gastrointestinal tract, breast, head, and neck in the clinic [8]. As a thymine antagonist possessing anticancer activities, 5-aminouracil (5AU) can block DNA synthesis and induce replication stress
$[10,11]$. In addition, recent findings indicate that microbiota can modulate the host response to these chemotherapeutic drugs [12]. Thus, the whole interactome of these fivesubstituted uracil drugs should be determined for detailed clinical pharmacokinetic and toxicity analyses.

Dihydropyrimidinase (DHPase) [13] is a key enzyme for pyrimidine degradation (Figure 1(a)). DHPase contains a binuclear metal center in which two $\mathrm{Zn}$ ions are bridged by a posttranslationally carbamylated lysine (Kcx) [14-18]. DHPase catalyzes the hydrolysis of dihydrouracil (DHU) to $N$-carbamoyl- $\beta$-alanine. Including a Kcx, the dimetal center $(\mathrm{Zn} \alpha / \mathrm{Zn} \beta)$ of DHPase consists of four His and one Asp. The conserved substrate binding sites of DHPase are Ser, Asn, and Tyr [19]. Of these residues, Ser and Asn interact with the 


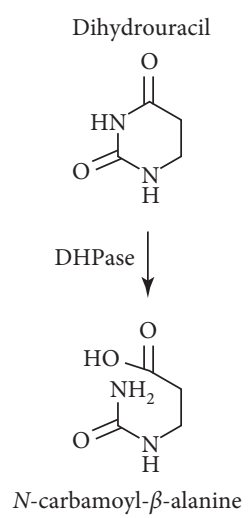

(a)

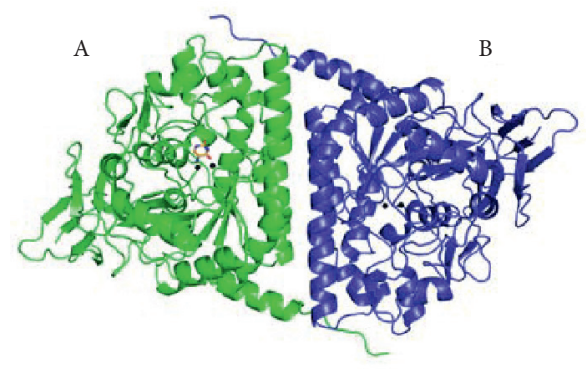

(b)

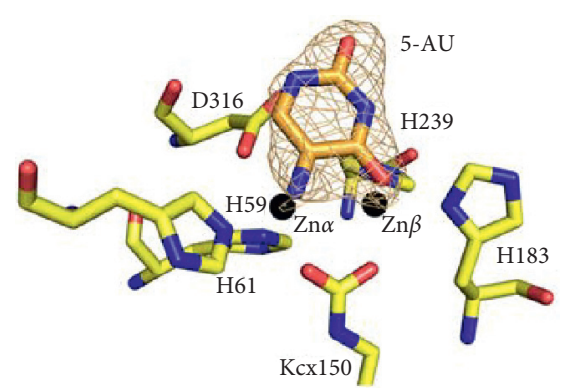

(c)

Figure 1: The structure of PaDHPase complexed with 5-AU. (a) The physiological reaction of DHPase. (b) The complexed structure of $\mathrm{PaDHPase}$ with 5-AU. The monomers are colored differently. 5-AU was found only in monomer A and not in monomer B. (c) The active site of PaDHPase. The binuclear metal center assembled by residues His59, His61, carbamylated Lys150 (Kcx150), His183, His239, and Asp316 in PaDHPase is essential for catalytic activity. The light orange mesh is the $2 \mathrm{Fo}-\mathrm{Fc}$ electron density map from the 5-AU contoured at $1 \sigma$ with a $1.6 \AA$ carve radius. Both two zinc ions were involved in binding 5-AU.

substrate DHU through the backbone of DHPase. Despite the interactions via the main chain, mutations of these residues still lead to severely impaired enzymes [16]. Loss of the $\mathrm{Zn} \beta$ ion causes $\mathrm{Kcx}$ to no longer be carbamylated in mono-Zn DHPase [14]. On the basis of an analysis of the amino acid sequences, DHPase was suggested to be a member of the cyclic amidohydrolase family $[20,21]$. The cyclic amidohydrolase family also includes dihydroorotase [22-28], allantoinase [28-30], hydantoinase [31, 32], and imidase [33-35]. These metal-dependent enzymes catalyze the hydrolysis of the cyclic amide bond of each substrate in the metabolism of purines and pyrimidines. Some of these amidohydrolases are known to be anticancer [24, 36-38], antimicrobial $[16,27,39]$, and antimalarial targets [40, 41] because of their involvement in the key reactions of nucleotide synthesis. Therefore, new inhibitors should be found, and their binding modes should be investigated for chemotherapeutic drug development.

5-FU-associated toxicity was reported [42]. After treatment of 5-FU for anticancer therapy, the asymptomatic patients with DHPase deficiency suffered from severe toxicity, including death [43]. A recent report revealed that DHPase is a novel 5-FU-binding protein [44]. Whether and how other five-substituted uracil drugs such as 5-AU can bind to DHPase remains to be elucidated. In the present study, we found that 5-AU can bind to Pseudomonas aeruginosa DHPase (PaDHPase). We determined the crystal structure of the PaDHPase complex with 5-AU at a $2.1 \AA$ resolution (PDB entry 7E3U). Given the structural resemblance between 5-FU and 5-AU, one might conclude that the mode of 5-FU bound by PaDHP must be similar to that of 5AU. However, we found that their binding modes are different. The fluorescence quenching method and site-directed mutagenesis were also used to confirm the binding mode of 5-AU to PaDHPase revealed by the complex structure. We think that the determination of this complex structure can extend the knowledge of the 5-AU interactome and benefit anticancer development.

\section{Materials and Methods}

2.1. Expression and Purification of PaDHPase. The expression vector pET21b-PaDHPase [16] was transformed into Escherichia coli BL21 (DE3) cells and grown in LB medium at $37^{\circ} \mathrm{C}$. The overexpression was induced by incubating with $1 \mathrm{mM}$ isopropyl thiogalactopyranoside for $9 \mathrm{~h}$. Recombinant PaDHPase [36] was purified from the soluble supernatant by using $\mathrm{Ni}^{2+}$-affinity chromatography (HiTrap HP; GE Healthcare Bio-Sciences). The recombinant protein was eluted with a linear imidazole gradient and dialyzed against a dialysis buffer $(20 \mathrm{mM}$ Tris- $\mathrm{HCl}$ and $0.1 \mathrm{M} \mathrm{NaCl}, \mathrm{pH}$ 7.9; Buffer A).

2.2. Preparation of Mono-Zn PaDHPase (Zno-PaDHPase). The mono- $\mathrm{Zn}$ PaDHPase was prepared using the protocol described previously [45]. Purified PaDHPase was dialyzed against a chelating buffer (50 mM MES, $50 \mathrm{mM}$ EDTA, and 15 mM 8-HQSA, pH 6.5; Buffer B) at room temperature for 3 days. The resultant enzyme solution was then dialyzed against Buffer A.

2.3. Site-Directed Mutagenesis. The PaDHPase mutants were generated according to the QuikChange Site-Directed Mutagenesis kit protocol (Stratagene; La Jolla, CA, USA). The wild-type plasmid pET21b-PaDHPase was used as a template [16]. The presence of the mutation was verified by DNA sequencing in each construct. The recombinant mutant proteins were purified using the protocol for the wildtype PaDHPase by $\mathrm{Ni}^{2+}$-affinity chromatography (Table 1 ).

2.4. Crystallography. The optimal protein concentration for crystallization of the PaDHPase complex, determined by the Pre-Crystallization Test (Hampton), was $10 \mathrm{mg} / \mathrm{mL}$. Crystals were grown at room temperature by hanging drop vapor diffusion in 16\% PEG 8000, $100 \mathrm{mM}$ HEPES, $200 \mathrm{mM}$ 
TABLe 1: Primers used for construction of plasmids.

\begin{tabular}{lc}
\hline Oligonucleotide & Primer \\
\hline Y155A-N & CACTTCATGGCCGCCAAGAACGCCATCATGGCC \\
Y155A-C & CATGATGGCGTTCTTGGCGGCCATGAAGTGCTT \\
S289A-N & GGCTACGTGATGGCCCCGCCGTTCCGTCCCGTC \\
S289A-C & GGGACGGAACGGCGGGGCCATCACGTAGCCGGC \\
C318A-N & CCGCCACCGACCACGCCTGCTTCTGCGCCGAGC \\
C318A-C & CGCAGAAGCAGGCGTGGTCGGTGGCGGTGGTAT \\
N337A-N & TTCAGCAAGATTCCCGCTGGCACGGCCGGCATC \\
N337A-C & GCCGGCCGTGCCAGCGGGAATCTTGCTGAAGTC \\
\hline
\end{tabular}

These plasmids were verified by DNA sequencing. Underlined nucleotides indicate the designated site for mutation or the restriction site.

calcium acetate, and $200 \mu \mathrm{M}$ 5-AU, at $\mathrm{pH}$ 7.5. Data were collected using an ADSC Quantum-315r CCD area detector at SPXF beamline BL13C1 at the National Synchrotron Radiation Research Center (NSRRC; Hsinchu, Taiwan). The structure of the 5-AU-PaDHPase complex was solved to $2.1 \AA$ resolution with the molecular replacement software Phaser-MR [46] using PaDHPase (PDB entry 5E5C) [15] as a model (Table 2). The data were indexed and scaled using HKL-2000 [47]. Models were built and refined with PHENIX [48] and Coot [49]. Coordinate and structure factor files have been deposited in the Protein Data Bank (PDB entry 7E3U).

2.5. Determination of the Dissociation Constant $\left(K_{d}\right)$. Through the fluorescence quenching analysis, the $K_{d}$ value of the purified PaDHPase was determined [44]. An aliquot of 5AU was added into the solution containing PaDHPase $(0.8 \mu \mathrm{M})$ and $50 \mathrm{mM}$ HEPES at $\mathrm{pH}$ 7.0. The decrease in the intrinsic fluorescence of PaDHPase was measured at $336 \mathrm{~nm}$ upon excitation at $279 \mathrm{~nm}$ and $25^{\circ} \mathrm{C}$ with a spectrofluorimeter (Hitachi F-2700; Hitachi High-Technologies, Japan). The $K_{\mathrm{d}}$ was obtained using the equation: $\Delta F=\Delta F_{\max }-K_{d}(\Delta F /[5-\mathrm{AU}])$.

\section{Results and Discussion}

3.1. Crystallization of the PaDHPase-5-AU Complex. $\mathrm{PaDHPase}$ crystals can be obtained by hanging drop vapor diffusion in 28\% PEG 6000, $100 \mathrm{mM}$ HEPES, and $200 \mathrm{mM}$ lithium acetate at $\mathrm{pH} 7.5$ [15]. Soaking and cocrystallization of PaDHPase with 5-AU under these conditions were attempted but were unsuccessful. After crystallization screening, the crystals of the PaDHPase-5-AU complex appeared at room temperature in 16\% PEG 8000, $100 \mathrm{mM}$ HEPES, $200 \mathrm{mM}$ calcium acetate, and $200 \mu \mathrm{M} 5-\mathrm{AU}$, at pH 7.5. These crystals were used for determining the complex structure of PaDHPase.

3.2. Structure of PaDHPase in Complex with 5-AU. The crystals of the 5-AU-PaDHPase complex belong to space group $\mathrm{P} 32_{1} 2_{1}$ with cell dimensions of $a=112.67, b=112.67$, and $c=161.43 \AA$. The complexed crystal structure of PaDHPase with 5-AU was determined at a $2.1 \AA$ resolution (Table 2). Two monomers of PaDHPase were found in the asymmetric unit (Figure 1(b)). Consistently, PaDHPase
TABLE 2: Data collection and refinement statistics.

\begin{tabular}{lc}
\hline Data collection & PaDHP-5AU \\
\hline Crystal & 1.0 \\
Wavelength $(\AA)$ & $30-2.16$ \\
Resolution $(\AA)$ & $\mathrm{P} 3_{1} 2_{1}$ \\
Space group & $112.67,161.43 / 90$ \\
Cell dimension $a, b, c(\AA) / \beta\left(^{\circ}\right)$ & $4.9(4.9)$ \\
Redundancy & $99.9(99.9)$ \\
Completeness $(\%)$ & $23.7(3.1)$ \\
$<I / \sigma I>$ & $0.965(0.866)$ \\
CC ${ }_{1 / 2}$ & \\
\hline Refinement & $29.01-2.16$ \\
Resolution $(\AA)$ & 64183 \\
No. of reflections & $0.185 / 0.229$ \\
$R_{\text {work }} / R_{\text {free }}$ & \\
No. of atoms & 1 \\
Ligands & 954 \\
Protein & 4 \\
Zinc & 459 \\
Water & \\
\hline r.m.s deviations & 0.008 \\
Bond lengths $(\AA)$ & 0.893 \\
Bond angles $\left({ }^{\circ}\right)$ & \\
\hline Ramachandran plot & 95.45 \\
Favored $(\%)$ & 3.7 \\
Allowed $(\%)$ & 0.85 \\
Outliers $(\%)$ & $7 \mathrm{E} 3 \mathrm{U}$ \\
PDB entry & \\
\hline Vales in parenthes & \\
\hline
\end{tabular}

Values in parentheses are for the highest resolution shell. $\mathrm{CC}_{1 / 2}$ is the percentage of correlation between the intensities of random half-data sets.

functions as a dimer [15]. The electron density of 5-AU was well defined and indicated the presence of 5-AU in the active site of PaDHPase (Figure 1(c)). The orientation of 5-AU could be easily distinguished on the basis of the location of the substituent. However, only one 5-AU molecule was found in the active site of one of the PaDHPase dimers. This is also the case for 5-FU bound to PaDHPase [44]. The binding of 5-AU does not influence the overall structure of PaDHPase. Similar to the apo form, the global architecture of the 5-AU-complexed PaDHPase monomer (subunit A) revealed a TIM-barrel structure embedding the catalytic dimetal center $(\mathrm{Zn} \alpha$ and $\mathrm{Zn} \beta)$ and a $\beta$-sandwich domain, consisting of $17 \alpha$-helices, $19 \beta$-sheets, $2 \mathrm{Zn}$ ions, and 15 -AU molecule. Both of the two zinc ions are involved in binding 5-AU. The dimetal center in the PaDHPase-5-AU complex 
consisted of His59, His61, Kcx150, His183, His239, and Asp316 still self-assembles (Figure 1(c)). Lys150 remains carbamylated (Kcx150) regardless of 5-AU binding.

3.3. 5-AU Binding Mode. As a uracil derivative possessing many chemotherapeutic and pharmacological activities [11], 5-AU was identified as a ligand bound by PaDHPase in this study. The 5-AU binding mode of PaDHPase was demonstrated by the complexed crystal structure. On the basis of our crystallographic analysis, various interactions between 5-AU and PaDHPase were examined (Figure 2(a)). Residues Tyr155, Ser289, and Asn337 of PaDHPase, crucial for substrate binding $[17,19]$, are also involved in 5-AU binding (Figure 2(b)). 5-AU interacts with $\mathrm{Zn} \alpha(3.2 \AA), \mathrm{Zn} \beta(3.0 \AA)$, the main chains of residues Ser289 (2.8 $\AA$ ) and Asn337 (3.3 $\AA$ ), and the side chain of residue Tyr155 (2.8 $\AA$ ). Dynamic loop I (amino acid residues Pro65-Val70) in PaDHPase is not involved in the binding of 5-AU.

3.4. Structural Comparison of the Active Sites between the 5$A U$ Bound State and the 5-FU Bound State of PaDHPase. Recently, the crystal structure of PaDHPase in complex with the anticancer drug 5-FU was reported [44]. 5-FU is the bestknown fluoropyrimidine used to target the enzyme thymidylate synthase in anticancer therapy $[3,8]$. Given the structural similarity between 5-AU and 5-FU (Figure 3(a)), one might conclude that their binding mode by PaDHPase must be similar. The dynamic loops of PaDHPase extend toward the active site when either 5-FU or 5-AU is bound (Figure 3(b)). However, we found that 5-AU and 5-FU binding poses to PaDHPase are different in terms of orientation (Figure 3(b)) and binding residues (Figure 3(c)). The side chain of residue Cys318 in PaDHPase (Figure 3(d)) is involved in binding 5-FU (2.9 $\AA$ ) but not 5-AU (Figure 2). In addition, the binding contributions from the metal ions in PaDHPase are different. $\mathrm{Zn} \beta$ is not involved in binding 5-FU [44], whereas both $\mathrm{Zn}$ ions interact with 5-AU (Figure 2). Thus, we concluded that the binding mechanisms of the anticancer drugs 5-FU and 5-AU to PaDHPase are different.

3.5. 5-AU Binding Analysis. Prior to this study, whether 5AU could bind to DHPase remained unknown. To confirm if PaDHPase is capable of binding 5-AU, we used the fluorescence quenching method to determine the binding ability of PaDHPase (Figure 4). Quenching is a complex formation process that decreases the fluorescence intensity of protein. The fluorescence emission spectra of the wild-type $\mathrm{PaDH}$ Pase was significantly quenched with 5-AU (Figure 4(a)). $\mathrm{PaDHPase}$ displayed strong intrinsic fluorescence with a peak wavelength of $336 \mathrm{~nm}$ when excited at $279 \mathrm{~nm}$. When 5-AU was titrated into the PaDHPase solution, the intrinsic fluorescence of the protein was progressively quenched. Upon the addition of $300 \mu \mathrm{M} 5-\mathrm{AU}$, the intrinsic fluorescence of PaDHPase was quenched by $87.3 \%$. Adding 5 -AU resulted in a red shift in the PaDHPase emission wavelength $\left(\sim 6.5 \mathrm{~nm} ; \lambda_{\max }\right.$ from 336.5 to $\left.343 \mathrm{~nm}\right)$. These observations indicated that PaDHPase can form a stable complex with 5-
AU. As determined through the titration curve, the dissociation constant $\left(K_{\mathrm{d}}\right)$ of PaDHPase bound to $5-\mathrm{AU}$ is $97.7 \pm 2.0 \mu \mathrm{M}$. Based on the $K_{\mathrm{d}}$ value of PaDHPase bound to 5-FU $(133.2 \pm 8.5 \mu \mathrm{M})$ [44], PaDHPase may prefer binding to 5-AU over 5-FU.

3.6. Structure-Based Mutational Analysis. The complexed structure revealed Tyr155, Ser289, and Asn337 of PaDHPase as the 5-AU binding sites (Figure 2). 5-AU interacts with the main chains of residues Ser289 and Asn337 and the side chain of residue Tyr155. To investigate the contribution of individual amino acid residues to 5-AU binding, alanine substitution mutants (Table 2) were constructed and analyzed by fluorescence quenching (Figures 4(b)-4(d)). We found that $300 \mu \mathrm{M}$ 5-AU quenched the intrinsic fluorescence of mutants Y155A, S289A, and N337A by $68.6 \%, 61.6 \%$, and $72.4 \%$, respectively. The $K_{\mathrm{d}}$ values of Y155A, S289A, and $\mathrm{N} 337 \mathrm{~A}$ bound to $5-\mathrm{AU}$ were reduced to $247.1 \pm 4.0$, $256.0 \pm 3.2$, and $192.1 \pm 8.0 \mu \mathrm{M}$, respectively. Accordingly, Ser289 is the most effective one of these residues for 5-AU binding.

Structurally, Cys318 is involved in 5-FU [44] but not 5AU. For comparison, the C318A mutant was also analyzed by fluorescence quenching (Figure 4(e)). We found that $300 \mu \mathrm{M} 5-\mathrm{AU}$ quenched the intrinsic fluorescence of C318A by $86.8 \%$. The $K_{\mathrm{d}}$ value of $\mathrm{C} 318 \mathrm{~A}$ was determined as $103.3 \pm 3.1 \mu \mathrm{M}$. The abilities of the wild-type PaDHPase and the $\mathrm{C} 318 \mathrm{~A}$ mutant to bind 5-AU are approximately equal. Thus, Cys318 is not essential for 5-AU binding.

3.7. Role of $Z n \beta$ Ion in 5-AU Binding. On the basis of the complex structure of 5-AU-PaDHPase, the $\mathrm{Zn} \beta$ ion is suggested to be critical for 5-AU binding (Figure 2). To confirm the role of $\mathrm{Zn} \beta$ in 5-AU binding and to compare the binding contribution with other mutant proteins (Table 3), we produced mono-Zn PaDHPase ( $\mathrm{Zn} \alpha$ PaDHPase) for binding analysis. Our crystal structure previously revealed that this mono-Zn enzyme [45] only contains a $\mathrm{Zn} \alpha$ ion in the active site of PaDHPase. The binding of 5-AU to $\mathrm{Zn} \alpha$-PaDHPase was also analyzed by the fluorescence quenching method (Figure 4(f)). Upon the addition of $300 \mu \mathrm{M} 5$-AU, the intrinsic fluorescence of $\mathrm{Zn} \alpha$-PaDHPase was quenched by $49.3 \%$. The $K_{\mathrm{d}}$ value of $\mathrm{Zn} \alpha$-PaDHPase for 5-AU binding was calculated to be $281.5 \pm 9.0 \mu \mathrm{M}$ from the titration curve (Figure 4(g)). Based on the $K_{\mathrm{d}}$ values, the strength of complex formation with 5-AU followed the following order: the wildtype enzyme $\approx \mathrm{C} 318 \mathrm{~A}>\mathrm{N} 337 \mathrm{~A}>\mathrm{Y} 155 \mathrm{~A} \approx \mathrm{S} 289 \mathrm{~A}>\mathrm{Zn} \alpha$ PaDHPase (Table 3).

3.8. The 5-AU Structural Interactome. Metabolic reprogramming allows the cancer cells to rapidly proliferate, resist chemotherapies, invade, metastasize, and survive in a nutrient-deprived microenvironment [1]. Many uracil derivatives have long been used for anticancer treatment, such as 5 -FU, the most commonly used pyrimidine-based antimetabolite targeting thymidylate synthase $[3,8]$. 5-AU also 


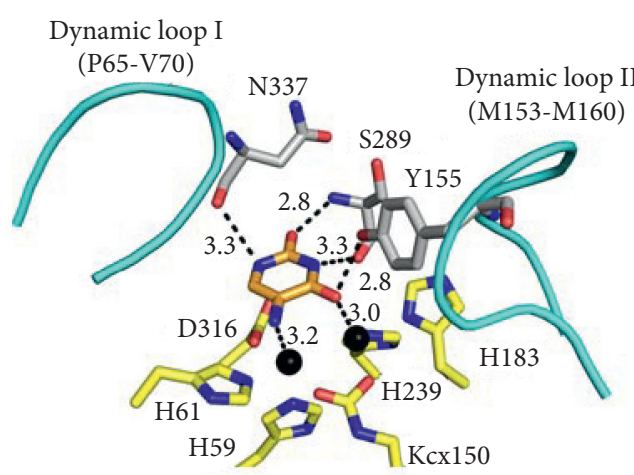

(a)

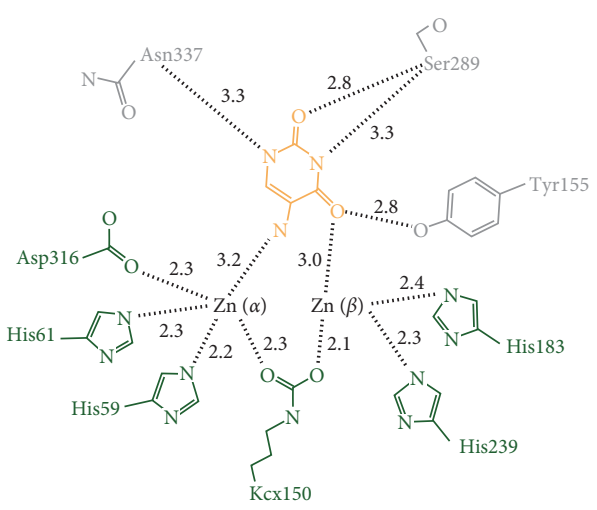

(b)

FIgURE 2: 5-AU binding mode. (a) The interactions of PaDHPase with 5-AU. Two metal ions (black spheres) and the substrate binding sites (gray) in PaDHPase were involved in 5-AU (light orange) binding. Dynamic loops are colored in cyan. 5-AU interacted with the main chains of residues Ser289 and Asn337 and the side chain of residue Tyr155. The distances are shown on dotted lines. (b) 5-AU interacted with Zn $\alpha$, Zn $\beta$, Ser289, Asn337, and Tyr155. Residues (His59, His61, Kcx150, His183, His239, and Asp316) required for metal binding are colored in green.<smiles>Nc1c[nH]c(=O)[nH]c1=O</smiles>

$5-\mathrm{AU}$<smiles>O=c1[nH]cc(F)c(=O)[nH]1</smiles>

5 -FU (a)

Dynamic loop I

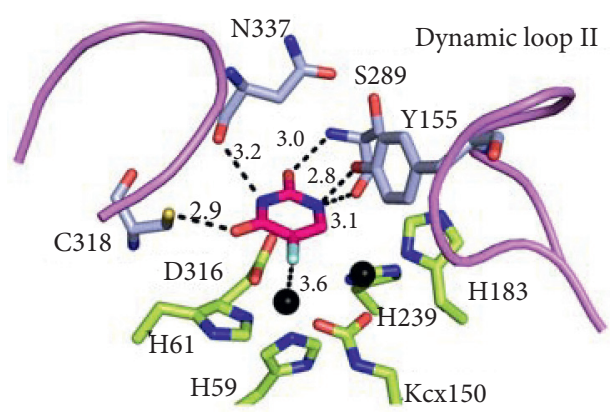

(c)
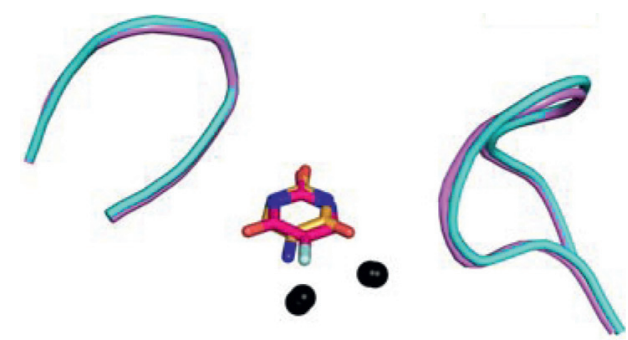

$-5-\mathrm{AU}$
$-5-\mathrm{FU}$ (b)

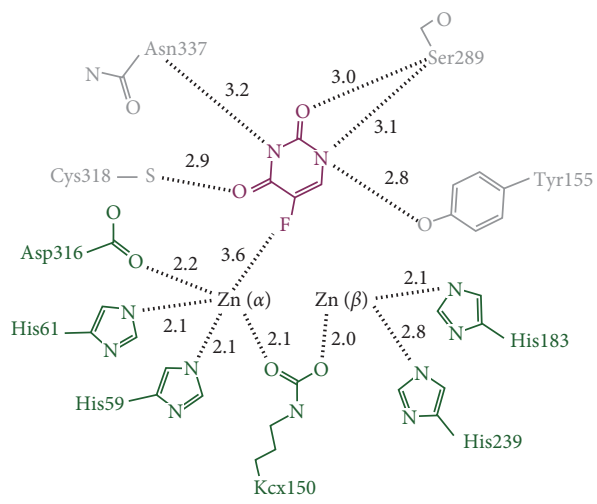

(d)

Figure 3: Comparison between the 5-AU and 5-FU binding modes. (a) The structure of 5-AU and 5-FU. (b) The superimposed structures of the 5-AU and 5-FU bound states. The conformation of the dynamic loops in both binding states is similar, but the orientations between 5-AU and 5-FU (hot pink) are different. (c) The interactions of PaDHPase with 5-FU. Zn $\alpha$ and the substrate binding sites (gray) in PaDHPase were involved in 5-FU binding. Dynamic loops are colored in light pink. 5-FU interacted with the main chains of residues Ser289 and Asn337 and the side chains of residues Tyr 155 and Cys318. The distances are shown on dotted lines. (d) 5-FU interacted with Zn $\alpha$, Ser289, Cys318, Asn337, and Tyr155. Residues (His59, His61, Kcx150, His183, His239, and Asp316) required for metal binding are colored in green.

possesses potent anticancer activities that can block DNA synthesis and induce replication stress [11]. 5-AU can significantly induce biphasic interphase-mitotic (IM) cells [10].
Although 5-AU and 5-FU are similar uracil derivatives, no induction of IM cells was detected even using excess 5-FU [10]. Therefore, 5-AU and 5-FU might somehow induce 


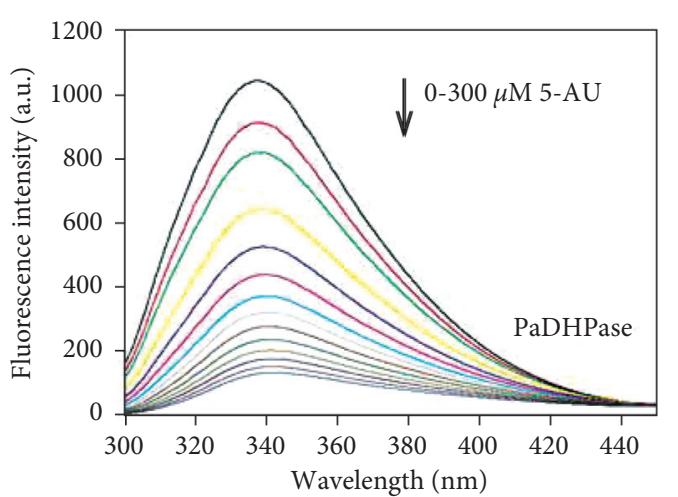

(a)

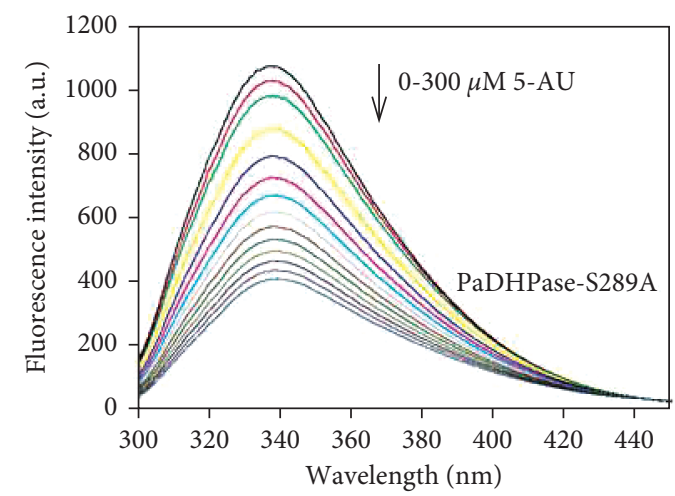

(c)

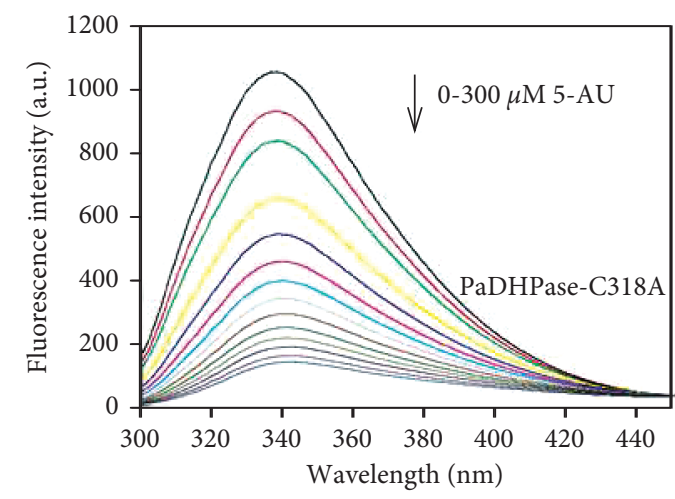

(e)

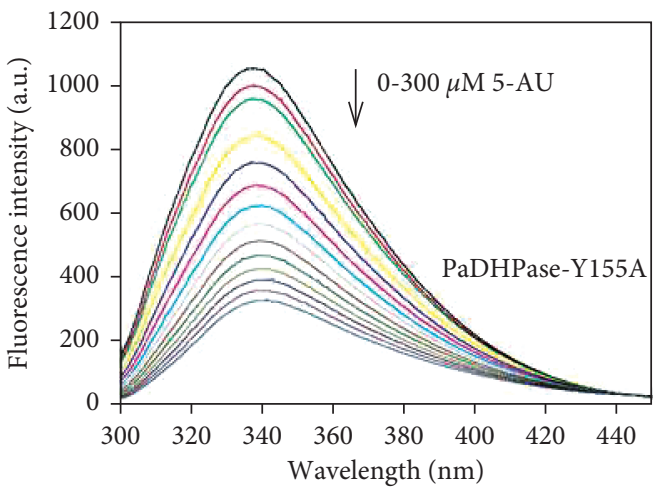

(b)

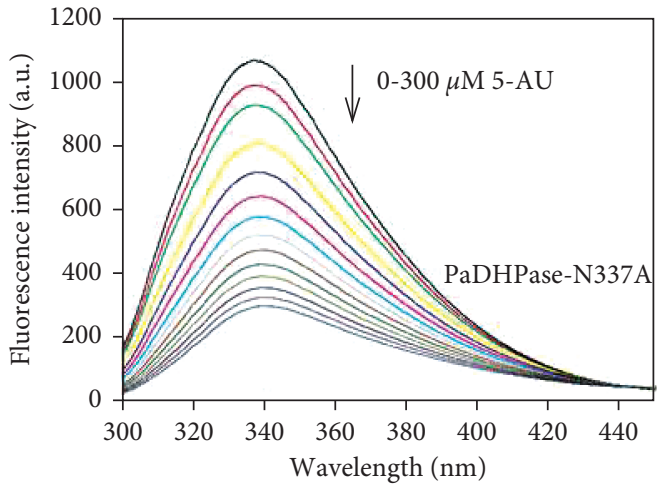

(d)

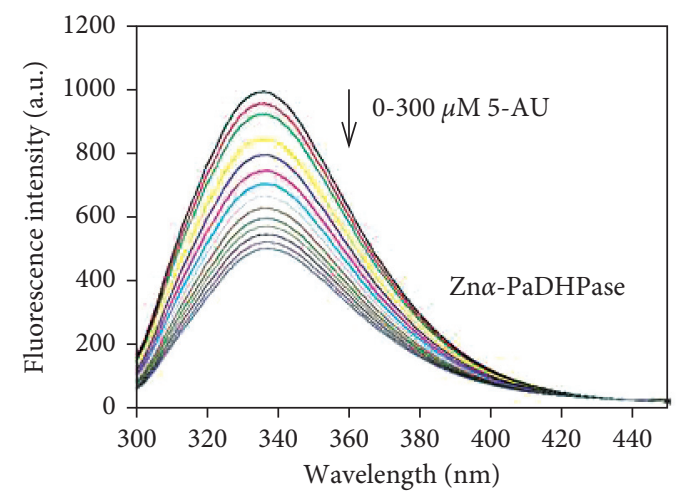

(f)

Figure 4: Continued. 


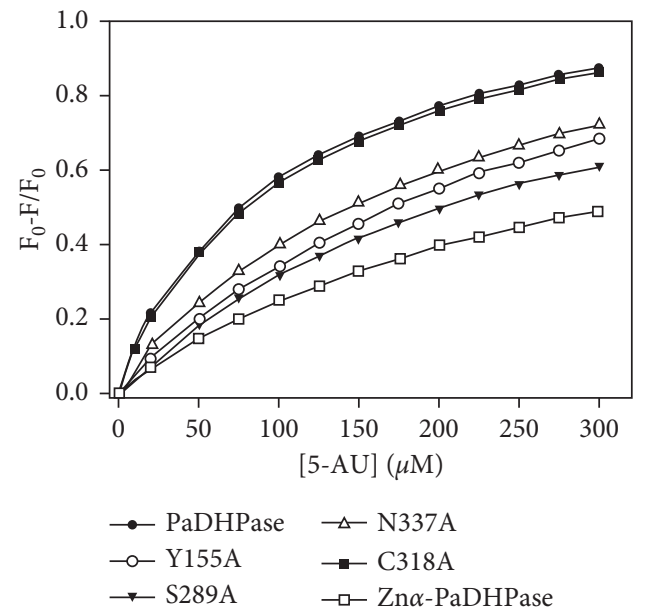

(g)

Figure 4: Fluorescence titration of PaDHPase with 5-AU. (a) The fluorescence emission spectra of PaDHPase with 5-AU of different concentrations $(0-300 \mu \mathrm{M})$. The decrease in intrinsic fluorescence of protein was measured at $336 \mathrm{~nm}$ upon excitation at $279 \mathrm{~nm}$ with a spectrofluorimeter. The fluorescence intensity emission spectra of PaDHPase significantly quenched with 5-AU. (b) The fluorescence emission spectra of PaDHPase-Y155A with 5-AU of different concentrations $(0-300 \mu \mathrm{M})$. (c) The fluorescence emission spectra of PaDHPase-S289A with 5-AU. (d) The fluorescence emission spectra of PaDHPase-N337A with 5-AU. (e) The fluorescence emission spectra of PaDHPase-C318A with 5-AU. (f) The fluorescence emission spectra of Zn $\alpha$-PaDHPase with 5-AU. (g) An aliquot amount of 5-AU was added to the enzyme solution for determining the $K_{\mathrm{d}}$. The $K_{\mathrm{d}}$ was obtained by the equation: $\Delta F=\Delta F_{\max }-K_{\mathrm{d}}(\Delta F /[5-\mathrm{AU}])$. Data points are an average of 2-3 determinations within $10 \%$ error.

TABle 3: Binding parameters of PaDHPase to 5-AU.

\begin{tabular}{lcccc}
\hline PaDHPase & $\lambda_{\max }(\mathrm{nm})$ & $\lambda_{\mathrm{em}}$ shift $(\mathrm{nm})$ & Quenching $(\%)$ & $K_{\mathrm{d}}$ value $(\mu \mathrm{M})$ \\
\hline PaDHPase & From 336.5 to 343 & 6.5 & 67.3 & $97.7 \pm 2.0$ \\
PaDHPase-Y155A & From 336.5 to 342 & 6.0 & 61.6 & $247.1 \pm 4.0$ \\
PaDHPase-S289A & From 337 to 339 & 2.0 & 72.4 & $256.0 \pm 3.2$ \\
PaDHPase-N337A & From 336 to 339.5 & 3.5 & 86.8 & $192.1 \pm 8.0$ \\
PaDHPase-C318A & From 336 to 342 & 6.0 & 49.3 & $103.3 \pm 3.1$ \\
$\mathrm{Zn} \alpha$-PaDHPase & From 335 to 336.5 & 1.5 & $281.5 \pm 9.0$ \\
\hline
\end{tabular}

The decrease in the intrinsic fluorescence of ScDHOase was measured with a spectrofluorimeter (Hitachi F-2700; Hitachi High-Technologies, Japan). The $K_{\mathrm{d}}$ was obtained using the equation: $\Delta F=\Delta F_{\max }-K_{\mathrm{d}}(\Delta F /[5-\mathrm{AU}])$.

The 5-AU dihydroorotase complex (PDB entry 6LOF)

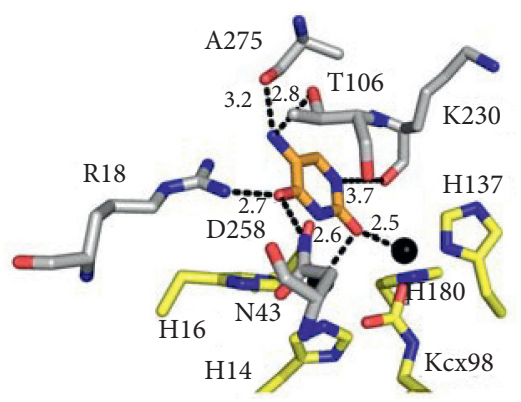

(a)
The 5-AU-uracil-DNA glycosylase complex (PDB entry 4WS6)

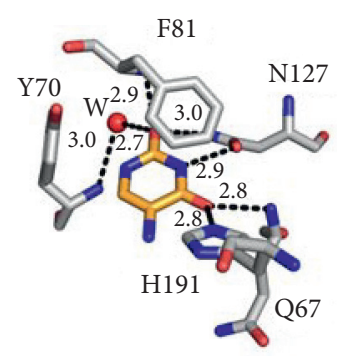

(b)

FIgURe 5: 5-AU binding modes. (a) The interactions of dihydroorotase with 5-AU. Two metal ions (black spheres) and the binding sites (gray) in dihydroorotase are involved in 5-AU (light orange) binding. (b) The interactions of uracil-DNA glycosylase with 5-AU.

different cellular effects. In this study, we found different binding modes of PaDHPase between 5-AU and 5-FU, and whether the different binding modes can produce distinct signaling pathways has not yet been elucidated. We noticed that other enzymes also respond to 5-AU by binding and/or inhibition. Three 5-AU-complexed protein structures are 
available in the PDB for comparison: DHPase (this study), uracil-DNA glycosylase (PDB entry 4WS6), and dihydroorotase (PDB entry 6L0F). These three enzymes bind 5$A U$ via different binding environments (Figure 5). For example, uracil-DNA glycosylase binds 5-AU via Gln67, Tyr70, Phe81, Asn127, and His191 [50]. Dihydroorotase binds 5-AU via Arg18, Asn43, Thr106, Lys230, and Ala275 [25]. These interactions involving 5-AU binding, including that of PaDHPase-5-AU, are different. Further structural studies are needed to understand 5-AU's binding mechanisms for building the structural interactome for detailed clinical pharmacokinetics and toxicity analyses.

\section{Conclusion}

We identified that PaDHPase can bind 5-AU with a $K_{\mathrm{d}}$ value of $97.7 \mu \mathrm{M}$ (Table 3 and Figure 4). The 5-AU binding mode of PaDHPase, different from that of 5-FU, was determined through structural evidence (Figure 1) and mutational analysis (Table 3 ). This structure provides molecular insights into how the dimetal center in PaDHPase can bind 5-AU (Figure 2). Further research can directly focus on revisiting the role of DHPase in anticancer therapy $[42,43,51]$.

\section{Data Availability}

Atomic coordinates and related structure factors were deposited in the PDB with accession code 7E3U. All the data used to support the findings of this study are available from the corresponding author upon request.

\section{Conflicts of Interest}

The authors declare that they have no conflicts of interest regarding the publication of this paper.

\section{Acknowledgments}

This research was supported by grants from the Ministry of Science and Technology, Taiwan (MOST 109-2622-E025-006 to E.S.L.) and Chung Shan Medical University (CSMU-INT-110-01 to C.Y.H.). The authors thank the experimental facility and the technical services provided by the Synchrotron Radiation Protein Crystallography Facility of the National Core Facility Program for Biotechnology, the Ministry of Science and Technology, Taiwan.

\section{References}

[1] J. Zhu and C. B. Thompson, "Metabolic regulation of cell growth and proliferation," Nature Reviews Molecular Cell Biology, vol. 20, no. 7, pp. 436-450, 2019.

[2] S. K. Fung and A. S. Lok, "Drug insight: nucleoside and nucleotide analog inhibitors for hepatitis B," Nature Clinical Practice Gastroenterology \& Hepatology, vol. 1, no. 2, pp. 90-97, 2004.

[3] D. B. Longley, D. P. Harkin, and P. G. Johnston, "5-Fluorouracil: mechanisms of action and clinical strategies," Nature Reviews Cancer, vol. 3, no. 5, pp. 330-338, 2003.

[4] A. E. J. Yssel, J. Vanderleyden, and H. P. Steenackers, "Repurposing of nucleoside- and nucleobase-derivative drugs as antibiotics and biofilm inhibitors," Journal of Antimicrobial Chemotherapy, vol. 72, no. 8, pp. 2156-2170, 2017.

[5] S.-K. Ku, M.-C. Baek, and J.-S. Bae, "Anti-inflammatory effects of methylthiouracil in vitro and in vivo," Toxicology and Applied Pharmacology, vol. 288, no. 3, pp. 374-386, 2015.

[6] M.-C. Baek, B. Jung, H. Kang, H.-S. Lee, and J.-S. Bae, "Novel insight into drug repositioning: methylthiouracil as a case in point," Pharmacological Research, vol. 99, pp. 185-193, 2015.

[7] H.-J. Lenz, S. Stintzing, and F. Loupakis, "TAS-102, a novel antitumor agent: a review of the mechanism of action," Cancer Treatment Reviews, vol. 41, no. 9, pp. 777-783, 2015.

[8] P. M. Wilson, P. V. Danenberg, P. G. Johnston, H.-J. Lenz, and R. D. Ladner, "Standing the test of time: targeting thymidylate biosynthesis in cancer therapy," Nature Reviews Clinical Oncology, vol. 11, no. 5, pp. 282-298, 2014.

[9] P. Álvarez, J. A. Marchal, H. Boulaiz et al., "5-Fluorouracil derivatives: a patent review," Expert Opinion on Therapeutic Patents, vol. 22, no. 2, pp. 107-123, 2012.

[10] A. Żabka, K. Winnicki, J. T. Polit, J. BernasińskaSłomczewska, and J. Maszewski, "5-Aminouracil and other inhibitors of DNA replication induce biphasic interphasemitotic cells in apical root meristems of Allium cepa," Plant Cell Reports, vol. 39, pp. 1013-1028, 2020.

[11] R. M. Shaker, M. A. Elrady, and K. U. Sadek, "Synthesis, reactivity, and biological activity of 5-aminouracil and its derivatives," Molecular Diversity, vol. 20, no. 1, pp. 153-183, 2016.

[12] J. L. Alexander, I. D. Wilson, J. Teare, J. R. Marchesi, J. K. Nicholson, and J. M. Kinross, "Gut microbiota modulation of chemotherapy efficacy and toxicity," Nature Reviews Gastroenterology \& Hepatology, vol. 14, no. 6, pp. 356-365, 2017.

[13] C.-Y. Huang, "Structure, catalytic mechanism, posttranslational lysine carbamylation, and inhibition of dihydropyrimidinases," Advances in Protein Chemistry and Structural Biology, vol. 122, pp. 63-96, 2020.

[14] J. H. Cheng, C. C. Huang, Y. H. Huang, and C. Y. Huang, "Structural basis for $\mathrm{pH}$-dependent oligomerization of dihydropyrimidinase from pseudomonas aeruginosa PAO1," Bioinorganic Chemistry and Applications, vol. 2018, Article ID 9564391, 8 pages, 2018.

[15] C.-T. Tzeng, Y.-H. Huang, and C.-Y. Huang, "Crystal structure of dihydropyrimidinase from Pseudomonas aeruginosa PAO1: insights into the molecular basis of formation of a dimer," Biochemical and Biophysical Research Communications, vol. 478, no. 3, pp. 1449-1455, 2016.

[16] C.-Y. Huang, "Inhibition of a putative dihydropyrimidinase from Pseudomonas aeruginosa PAO1 by flavonoids and substrates of cyclic amidohydrolases," PLoS One, vol. 10, no. 5, Article ID e0127634, 2015.

[17] Y.-C. Hsieh, M.-C. Chen, C.-C. Hsu, S. I. Chan, Y.-S. Yang, and C.-J. Chen, "Crystal structures of vertebrate dihydropyrimidinase and complexes from Tetraodon nigroviridis with lysine carbamylation," Journal of Biological Chemistry, vol. 288, no. 42, pp. 30645-30658, 2013.

[18] J. Abendroth, K. Niefind, and D. Schomburg, "X-ray structure of a dihydropyrimidinase from thermus sp. at $1.3 \AA$ resolution," Journal of Molecular Biology, vol. 320, no. 1, pp. 143-156, 2002.

[19] B. Lohkamp, B. Andersen, J. Piškur, and D. Dobritzsch, "The crystal structures of dihydropyrimidinases reaffirm the close relationship between cyclic amidohydrolases and explain their 
substrate specificity," Journal of Biological Chemistry, vol. 281, no. 19, pp. 13762-13776, 2006.

[20] J. A. Gerlt and P. C. Babbitt, "Divergent evolution of enzymatic function: mechanistically diverse superfamilies and functionally distinct suprafamilies," Annual Review of Biochemistry, vol. 70, no. 1, pp. 209-246, 2001.

[21] G.-J. Kim and H.-S. Kim, "Identification of the structural similarity in the functionally related amidohydrolases acting on the cyclic amide ring," Biochemical Journal, vol. 330, no. 1, pp. 295-302, 1998.

[22] A. J. Rice, R. P. Pesavento, J. Ren et al., "Identification of small molecule inhibitors against Staphylococcus aureus dihydroorotase via HTS," International Journal of Molecular Sciences, vol. 22, no. 18, p. 9984, 2021.

[23] H. H. Guan, Y. H. Huang, E. S. Lin, C. J. . Chen, and C. Y. Huang, "Complexed crystal structure of Saccharomyces cerevisiae dihydroorotase with inhibitor 5-fluoroorotate reveals a new binding mode," Bioinorganic Chemistry and Applications, vol. 2021, Article ID 2572844, 9 pages, 2021.

[24] H.-H. Guan, Y.-H. Huang, E.-S. Lin, C.-J. Chen, and C.-Y. Huang, "Plumbagin, a natural product with potent anticancer activities, binds to and inhibits dihydroorotase, a key enzyme in pyrimidine biosynthesis," International Journal of Molecular Sciences, vol. 22, no. 13, p. 6861, 2021.

[25] H.-H. Guan, Y.-H. Huang, E.-S. Lin, C.-J. Chen, and C.-Y. Huang, "Structural basis for the interaction modes of dihydroorotase with the anticancer drugs 5-fluorouracil and 5-aminouracil," Biochemical and Biophysical Research Communications, vol. 551, pp. 33-37, 2021.

[26] F. Del Caño-Ochoa and S. Ramón-Maiques, "Deciphering CAD: structure and function of a mega-enzymatic pyrimidine factory in health and disease," Protein Science, vol. 30, pp. 1995-2008, 2021.

[27] W.-F. Peng and C.-Y. Huang, "Allantoinase and dihydroorotase binding and inhibition by flavonols and the substrates of cyclic amidohydrolases," Biochimie, vol. 101, pp. 113-122, 2014.

[28] Y.-Y. Ho, Y.-H. Huang, and C.-Y. Huang, "Chemical rescue of the post-translationally carboxylated lysine mutant of allantoinase and dihydroorotase by metal ions and short-chain carboxylic acids," Amino Acids, vol. 44, no. 4, pp. 1181-1191, 2013.

[29] Y.-Y. Ho, H.-C. Hsieh, and C.-Y. Huang, "Biochemical characterization of allantoinase from Escherichia coli BL21," The Protein Journal, vol. 30, no. 6, pp. 384-394, 2011.

[30] K. Kim, M.-I. Kim, J. Chung, J.-H. Ahn, and S. Rhee, "Crystal structure of metal-dependent allantoinase from Escherichia coli," Journal of Molecular Biology, vol. 387, no. 5, pp. 1067-1074, 2009.

[31] C.-Y. Huang, C.-C. Hsu, M.-C. Chen, and Y.-S. Yang, "Effect of metal binding and posttranslational lysine carboxylation on the activity of recombinant hydantoinase," JBIC Journal of Biological Inorganic Chemistry, vol. 14, no. 1, pp. 111-121, 2009.

[32] Z. Xu, Y. Liu, Y. Yang, W. Jiang, E. Arnold, and J. Ding, "Crystal structure of d -hydantoinase from burkholderia pickettii at a resolution of 2.7 angstroms: insights into the molecular basis of enzyme thermostability," Journal of Bacteriology, vol. 185, no. 14, pp. 4038-4049, 2003.

[33] C.-Y. Huang and Y.-S. Yang, "A novel cold-adapted imidase from fish Oreochromis niloticus that catalyzes hydrolysis of maleimide," Biochemical and Biophysical Research Communications, vol. 312, no. 2, pp. 467-472, 2003.
[34] C.-Y. Huang and Y.-S. Yang, "The role of metal on imide hydrolysis: metal content and $\mathrm{pH}$ profiles of metal ionreplaced mammalian imidase," Biochemical and Biophysical Research Communications, vol. 297, no. 4, pp. 1027-1032, 2002.

[35] Y. S. Yang, S. Ramaswamy, and W. B. Jakoby, "Rat liver imidase," Journal of Biological Chemistry, vol. 268, no. 15, pp. 10870-10875, 1993.

[36] Y.-H. Huang, Y. Lien, J.-H. Chen, E.-S. Lin, and C.-Y. Huang, "Identification and characterization of dihydropyrimidinase inhibited by plumbagin isolated from Nepenthes miranda extract," Biochimie, vol. 171-172, pp. 124-135, 2020.

[37] J. S. Lee, L. Adler, H. Karathia et al., "Urea cycle dysregulation generates clinically relevant genomic and biochemical signatures," Cell, vol. 174, no. 6, pp. 1559-1570, 2018.

[38] S. Rabinovich, L. Adler, K. Yizhak et al., "Diversion of aspartate in ASS1-deficient tumours fosters de novo pyrimidine synthesis," Nature, vol. 527, no. 7578, pp. 379-383, 2015.

[39] E. R. Verrier, A. Weiss, C. Bach et al., "Combined small molecule and loss-of-function screen uncovers estrogen receptor alpha and CAD as host factors for HDV infection and antiviral targets," Gut, vol. 69, no. 1, pp. 158-167, 2019.

[40] M. Lee, C. W. Chan, S. C. Graham, R. I. Christopherson, J. M. Guss, and M. J. Maher, "Structures of ligand-free and inhibitor complexes of dihydroorotase from Escherichia coli: implications for loop movement in inhibitor design," Journal of Molecular Biology, vol. 370, no. 5, pp. 812-825, 2007.

[41] K. K. Seymour, S. D. Lyons, L. Phillips, K. H. Rieckmann, and R. I. Christopherson, "Cytotoxic effects of inhibitors of de novo pyrimidine biosynthesis upon Plasmodium falciparum," Biochemistry, vol. 33, no. 17, pp. 5268-5274, 1994.

[42] S. Sumi, M. Imaeda, K. Kidouchi et al., "Population and family studies of dihydropyrimidinuria: prevalence, inheritance mode, and risk of fluorouracil toxicity," American Journal of Medical Genetics, vol. 78, no. 4, pp. 336-340, 1998.

[43] A. B. van Kuilenburg, R. Meinsma, B. A. Zonnenberg et al., "Dihydropyrimidinase deficiency and severe 5-fluorouracil toxicity," Clinical Cancer Research, vol. 9, pp. 4363-4367, 2003.

[44] Y.-H. Huang, Z.-J. Ning, and C.-Y. Huang, "Crystal structure of dihydropyrimidinase in complex with anticancer drug 5fluorouracil," Biochemical and Biophysical Research Communications, vol. 519, no. 1, pp. 160-165, 2019.

[45] J.-H. Cheng, Y.-H. Huang, J.-J. Lin, and C.-Y. Huang, "Crystal structures of monometallic dihydropyrimidinase and the human dihydroorotase domain K1556A mutant reveal no lysine carbamylation within the active site," Biochemical and Biophysical Research Communications, vol. 505, no. 2, pp. 439-444, 2018.

[46] A. J. McCoy, R. W. Grosse-Kunstleve, P. D. Adams, M. D. Winn, L. C. Storoni, and R. J. Read, "Phasercrystallographic software," Journal of Applied Crystallography, vol. 40, no. 4, pp. 658-674, 2007.

[47] Z. Otwinowski and W. Minor, "Processing of X-ray diffraction data collected in oscillation mode," Methods in Enzymology, vol. 276, pp. 307-326, 1997.

[48] J. J. Headd, N. Echols, P. V. Afonine et al., "Use of knowledge-based restraints in phenix.refine to improve macromolecular refinement at low resolution," Acta Crystallographica Section D Biological Crystallography, vol. 68, no. 4, pp. 381-390, 2012. 
[49] P. Emsley and K. Cowtan, "Coot: model-building tools for molecular graphics," Acta Crystallographica Section D Biological Crystallography, vol. 60, no. 12, pp. 2126-2132, 2004.

[50] S. M. Arif, K. Geethanandan, P. Mishra, A. Surolia, U. Varshney, and M. Vijayan, "Structural plasticity in $M y$ cobacterium tuberculosis uracil-DNA glycosylase (MtUng) and its functional implications," Acta Crystallographica. Section D, Biological Crystallography, vol. 71, pp. 1514-1527, 2015.

[51] G. D. Heggie, J. P. Sommadossi, D. S. Cross, W. J. Huster, and R. B. Diasio, "Clinical pharmacokinetics of 5-fluorouracil and its metabolites in plasma, urine, and bile," Cancer Research, vol. 47, pp. 2203-2206, 1987. 